Clinical Medicine

Poster

Abstract ID: 59

\title{
Success rate of endodontically treated patients attending polyclinic, Kulliyyah of Dentistry, International Islamic University Malaysia
}

Nazih Shaban Mustafa | Muhannad Ali Kashmoola | Omar Abdul Jabbar

Department of Oral Medicine and Oral Pathology, Kulliyyah of Dentistry, International Islamic University Malaysia

Introduction: The aim of this study is to determine the success rate of the endodontically treated teeth in patients attending the Polyclinic, Kulliyyah of Dentistry IIUM from 2012 to 2015. Methods: A retrospective study involved endodontically treated teeth of patients attending the Polyclinic, Kulliyyah of Dentistry IIUM from 2012 to 2015. Clinical and radiographic data were recorded and classified as successful or failed, and further analyzed by Fisher's exact test to measure the correlation between the variables by using SPSS software version 16.0. Kappa test was used to measure the overall relationship between clinical and radiographic findings. Results: The overall success rate of the endodontically treated teeth is $85 \%(n=51)$. Correlation between the variables showed no significant $(p>0.05)$ in the success rate among age groups, between gender, diagnosis and race at the time of treatment, between upper and lower arches, and between anterior and posterior tooth. Correlation between the variable of post-endodontic fixed restorations showed statistically significant in the success rate $(p<0.05)$. Conclusions: High success rate $85 \%$ among patients with no signs and symptoms and with no radiographic changes of post-endodontic fixed restorations. Age, gender and race have no significant relations on the success rate of endodontically treated teeth.

KEYWORDS: Endodontic, Success rate, restoration, anterior teeth 